\title{
L'esame microbiologico dei campioni di feci: procedura operativa diagnostica
}

\section{Daniele Crotti', Maria Letizia D'Annibale², Alberto Catania ${ }^{3}$, Giacomo Magnani ${ }^{3}$}

'Libero Professionista in Parassitologia e Microbiologia Medica, Perugia

${ }^{2}$ Struttura Complessa di Microbiologia, Azienda Ospedaliera, Perugia

${ }^{3}$ Divisione di Malattie Infettive, Azienda Ospedaliera Ospedale S. Maria Nuova, Reggio Emilia

\section{Infectious diarrhoea: proposal of cost-effective diagnostic procedures}

PREMESSE

Batteri associati alle infezioni intestinali o gastrointestinali

Diarrea associata ad antibiotici

Batteri implicati in tossinfezioni alimentari

Agenti virali

PROCEDURE DIAGNOSTICHE

Diagnostica di base

Diagnostica allargata

- diarrea acuta autoctona

- diarrea protratta

- diarrea nel paziente immunocompromesso

- diarrea del viaggiatore

- enterite epidemica in collettività

\section{PREMESSE}

Le infezioni intestinali diarroiche rimangono ancora oggi la causa principale di morbilità e mortalità diffusa nel mondo nonostante i progressi nella comprensione della loro epidemiologia, patogenesi e clinica.

In tale Procedura Operativa Diagnostica si analizzano gli agenti patogeni batterici e virali, che, a livello intestinale, sono responsabili della diarrea infettiva.

Per la ricerca di protozoi (o altri parassiti), responsabili di enteriti, è opportuno consultare le "Linee Guida Operative per la diagnosi delle Parassitosi Intestinali" già pubblicate su Microbiologia Medica 2005; 20(1): 39-46.

La diarrea acuta è definita come una patologia di durata inferiore alle 2 settimane. Per diarrea infettiva (e quindi enterite) si intende l'emissione di feci liquide o quantomeno non formate per quattro o più volte al giorno, associata solitamente ad almeno uno dei seguenti segni o sintomi: dolori addominali, nausea, vomito, malessere generale, febbre, disidratazione (diarrea moderata o severa). Si definisce diarrea lieve se non si associa a sintomi addominali o sistemici. Le feci possono essere acquose o dissenteriche e contenere un aumento di leucociti, o essere mucose e/o ematiche in modo evidente od occulto.

I meccanismi patogenetici batterici di base prevedono:
- enterocolite emorragica

- enterocolite da antibiotici/iatrogena/in pazienti ospedalizzati

- proctocoliti nel maschio omosessuale

- tossinfezioni alimentari

- situazioni particolari

RACCOLTA, CONSERVAZIONE ED INVIO DEI CAMPIONI FECALI PROCEDURE DIAGNOSTICHE PRELIMINARI TERRENI DI COLTURA, MICROORGANISMI E MODALITÀ OPERATIVE IDENTIFICAZIONI PROCEDURE DI REFERTAZIONE Bibliografia

a) la resistenza all'eliminazione tramite $i$ fattori di adesione (E. coli: EAEC ed EPEC);

b) l'invasione della mucosa (Shigella, Salmonella, Campylobacter ed E. coli EIEC);

c) la produzione di enterotossine (vibrioni ed $E$. coli enterotossigeno);

d) la produzione di citotossine (Shigella dysenteriae ed E. coli VTEC [O-157, ad esempio]).

Tra i fattori protettivi ricordiamo la barriera acida gastrica e la fisiologica popolazione batterica intestinale.

Questa procedura raccomanda la ricerca routinaria, in campioni di feci non solide (non formate o poltacee, liquide, acquose), di Salmonella, Shigella e Campylobacter (COPROCOLTURA: diagnostica minima).

Le ricerche colturali di Escherichia coli enteritogeni, Yersinia enterocolitica, Vibrio choleare e/o altri vibrioni (Vibrio, Aeromonas, Plesiomonas), la ricerca di Clostridium difficile, la ricerca di Rotavirus e Adenovirus, sono da intendersi come complementari o supplementari, quando motivate (diagnostica allargata).

Batteri associati alle infezioni intestinali o gastro-intestinali

\section{Salmonella}

Le infezioni da Salmonella enterica (salmonelle minori), bastoncello Gram negativo mobile, di cui 
sono noti ben oltre 2000 sierotipi (o sottospecie o serovars), hanno un'incubazione usualmente variabile da 12 a 48 ore e sono ampiamente distribuite tra vari generi e specie animali. Le più diffuse sono attualmente $S$. typhimurium e $S$. enteritidis. Le salmonelle maggiori, quali $S$. typhi e $S$. paratyphi (gruppo A e $\mathrm{B}$, raramente $\mathrm{C}$ ), responsabili delle febbri tifoidee e paratifoidee, vanno ricercate soprattutto in emocolture, in quanto responsabili di sepsi ed essendo l'isolamento dalle feci raro, casuale od osservabile solo in una fase tardiva del quadro clinico.

La trasmissione è, come in tutte le infezioni intestinali, fecale-orale. Nell'uomo l'infezione si contrae per ingestione di acqua infetta o alimenti (uova o carni crude/non cotte/mal cotte [ma Salmonella si replica anche negli alimenti malconservati]) contaminati, oppure per contatto orale con dita contaminate con materiale fecale. Il quadro clinico è estremamente variabile.

In ambienti chiusi sono possibili episodi epidemici ( 2 o più individui sono colpiti), anche per trasmissione diretta da persona a persona, a causa di mancato rispetto di adeguate norme igieniche.

Tra le forme a eziologia batterica, le enteriti da Salmonella sono in Italia tuttora le più frequenti, seppur non dovunque.

\section{Shigella}

Si conoscono quattro specie di Shigella: S. sonnei, S. flexneri, S. dysenteriae, S. boydii. È un bastoncello Gram-negativo, immobile, ad alta contagiosità (bastano 200 germi) con incubazione breve (24-48 h).

In Italia Shigella è piuttosto rara, e solitamente è isolata S. sonnei.

S. dysenteriae (assai raramente altre) può causare grave dissenteria; febbre è sovente concomitante, ma mai nelle infezioni da $S$. sonnei.

Le shigellosi sono prettamente umane, e i microrganismi si trasmettono da persona a persona attraverso le dita, per via idro-alimentare, da fomiti contaminati (la dose infettante è bassa, laddove per Salmonella è elevata).

In comunità chiuse, sovraffollate, a bassi standard igienici (prigioni, scuole, istituzioni per malati mentali, e simili), sono possibili epidemie, anche con decessi per disidratazione.

\section{Campylobacter}

In Italia prevalgono i ceppi termotolleranti di Campylobacter jejuni e, più di rado, Campylobacter coli. Le campylobacteriosi sono seconde, come frequenza, alle salmonellosi, seppure in alcune aree geografiche di Paesi sviluppati possono essere prevalenti. Raramente è presente Arcobacter butzleri, genere correlato a
Campylobacter.

Alcuni ceppi sono responsabili di enteriti invasive, con muco e sangue; alcuni sierotipi di C. jeju$n i$ (in particolare 1'0:19) sono correlati a complicanze quali la Sindrome di Guillain-Barrè. Talora si associano quadri simulanti un'appendicite.

Campylobacter alberga l'intestino di svariati animali (anche esotici), e la trasmissione è fecaleorale, analogamente a Salmonella, anche se è soprattutto il pollame la causa principale di infezione umana (la dose infettante è relativamente bassa, e i microrganismi non si replicano sugli alimenti).

\section{Escherichia coli}

Le infezioni intestinali sostenute da $E$. coli cosiddetti enteritogeni sono probabilmente piuttosto infrequenti in Italia, e usualmente non gravi. Il periodo d'incubazione varia da $24-72 \mathrm{~h}$.

Si conoscono i seguenti "gruppi": ETEC, EPEC, EIEC, VTEC (tra cui l'EHEC 0:157), EAEC ed EaggEC.

E. coli-ETEC (enterotossigenico) è causa principale di diarrea del viaggiatore, mostrando quadri clinici variabili, ma in genere autorisolventesi.

E. coli - EPEC (enteropatogeno) può causare diarrea, anche epidemica, in bambini sotto i 3 anni di vita; sembra però essere piuttosto raro.

E. coli - EIEC (enteroinvasivo) causa un'enterite simildissenterica, anche severa, in Paesi in via di sviluppo; i ceppi responsabili possono essere confusi con Shigella.

E. coli - VTEC (verocitotossico) causa anche colite emorragica. Il sierotipo 0:157 può causare la Sindrome Uremico-Emolitica. In Italia è stato eccezionalmente descritto e sembra essere correlato soprattutto all'ingestione di carne bovina macinata malcotta. Vi possono essere episodi epidemici anche perché la dose infettante è bassa.

E. coli - EaggEC (enteroaggregante) ed E. coliEAEC (enteroadesivo) causa diarrea di entità variabile in alcune parti del mondo; non sembra essere stato rilevato in Italia.

\section{Yersinia enterocolitica}

L'infezione da Yersinia enterocolitica, bastoncello Gram-negativo e sempre appartenente alla Famiglia delle Enterobacteriaceae, è rara in Italia. I sierotipi implicati sono essenzialmente 1'0:3 e 1'0:9. Le enteriti acute con diarrea causate da $Y$. enterocolitica solitamente non sono gravi, e possono essere accompagnate da faringite. In alcune aree geografiche italiane, i pazienti solitamente talassemici in terapia con chelanti del ferro possono sviluppare una diarrea da $Y$. enterocolitica. L'infezione è di solito acquisita per ingestione di alimenti, acqua o latte contaminati da feci animali (il suino è spesso identificato quale sorgente di 
infezione).

Le yersiniosi, comunque assai rare, possono decorrere con quadri clinici diversificati: linfadenite mesenterica, ileite terminale, pseudoappendicite, e possono residuare in sequele immunologiche (quale l'artrite reattiva), più spesso delle campylobacteriosi e delle shigellosi.

\section{Vibrio spp.}

Le specie enteropatogene di Vibrio possono causare sintomi che vanno da forme lievi a diarree ematiche; talora il quadro clinico è assai grave ( $\mathrm{si}$ pensi al colera). L'incubazione è fra 6 e $72 \mathrm{~h}$.

Le principali specie patogene sono Vibrio cholerae (0:1 e 0:139), assente in Italia, e $V$. parahaemolyticus (assai raro se non eccezionale in Italia ma molto diffuso, per esempio, in Giappone). Sono bastoncelli ricurvi Gram-negativi e tipicamente alofili d'acqua marina.

I principali sintomi del colera sono rappresentati dal transito profuso di diarrea acquosa con muco ma senza sangue, conferendo un aspetto "ad acqua di riso", con l'alto rischio di perdite di liquidi, disidratazione e decesso. Il colera si presenta solitamente epidemico, ed è associato soprattutto ad acque contaminate. $V$. cholerae non 0:1/0:139 solitamente non è produttore della enterotossina responsabile della severità del quadro clinico.

$V$. parahaemolyticus è causa di gastroenteriti conseguenti di solito ad ingestione di frutti di mare o pesci non cotti (ma anche ingestione di acqua contaminata).

\section{Aeromonas spp.}

Le specie di Aeromonas, e, soprattutto A. hydrophila, sono state implicate quali cause di diarree non gravi, soprattutto in pazienti anziani. In Italia A. hydrophila è rara.

In genere l'infezione è conseguenza di contaminazioni idriche.

\section{Plesiomonas shigelloides}

In Italia, $P$. shigelloides sembra essere pressoché assente. Presenta analogie con $A$. hydrophila, e il suo ruolo è in parte controverso (al pari di Aeromonas).

\section{Clostridium difficile}

Pur essendo causa di diarrea in ambiente comunitario, come, e forse ancora prima in ambiente ospedaliero, in soggetti non in terapia antibiotica, C. difficile è soprattutto correlabile alla diarrea associata agli antibiotici (vedi oltre).

\section{Diarrea associata ad antibiotici}

\section{Clostridium difficile}

C. difficile è il patogeno più frequentemente isolato da pazienti con diarrea associata a terapia con antibiotici. Quando la popolazione microbica del tratto intestinale è alterata, $C$. difficile può moltiplicarsi in modo rigoglioso, con la successiva produzione di tossine (enterotossina di tipo A e citotossina di tipo B). La tossina determina danni caratteristici della mucosa consistenti in lesioni a forma di placca che portano alla formazione delle pseudomembrane. Comunque non tutti i ceppi sono produttori di tossine ed inducono malattia.

La maggior parte dei farmaci ad ampio spettro di attività è stata implicata come agente causale di diarrea associata agli antibiotici. I più frequentemente implicati sono clindamicina, ampicillina e simili, cefalosporine.

La malattia ha uno spettro che varia da diarrea autolimitante di moderata entità a progressive e gravi forme caratteristiche di colite pseudomembranosa.

La diagnosi più accurata di colite pseudomembranosa è ottenuta con il rilievo endoscopico delle pseudomembrane o dei microascessi del colon nei pazienti trattati con antibiotici che presentano diarrea e le cui feci sono positive per $C$. difficile e la sua tossina.

La percentuale di colonizzazione dei pazienti ospedalizzati può superare il 20\%. Il batterio può essere associato ad epidemie ospedaliere (ne è un'importante causa), così come in grosse strutture di ricovero per anziani. $C$. difficile può essere isolato dall'ambiente ospedaliero, e può essere riscontrato sui pavimenti, nei servizi igienici, sugli effetti personali intimi e letterecci. Per tale motivo è indicato l'isolamento nell'individuo malato ospedalizzato.

La procedura diagnostica di riferimento è la coltura cellulare; peraltro si raccomanda solitamente la ricerca della tossina A e B con metodi ELISA (o comunque immunologici).

\section{Batteri implicati in tossinfezioni alimentari}

Sono rappresentati da Staphylococcus aureus (produttore di enterotossina termostabile), Bacillus cereus (forma emetica e forma diarroica), Clostridium perfringens (ingestione usualmente della tossina A).

In tale procedura vengono esclusi il botulismo e altre più eccezionali patologie come quelle da ingestioni di tossine in molluschi, pesci equatoriali ed anfibi.

Le tossinfezioni alimentari sono in Italia molto più rare del passato. Per definizione dovrebbero essere dette tali quelle gastroenteriti, con nausea, vomito e dolori addominali (e poi diarrea), senza febbre, legate all'ingestione di tossine (enterotossine) con gli alimenti contaminati (sovente vere e 
proprie intossicazioni). A brevissima (entro 6 ore) o a breve incubazione (entro 12-24 ore), colpiscono varie persone, dopo pasti consumati in comune. S. aureus è in causa soprattutto nella ingestione di prodotti di pasticceria (a base di latte e/o uova preparati in casa, ma non solo); B. cereus nella ingestione di riso fritto o di creme alla vaniglia; C. perfringens nella ingestione soprattutto di alimenti carnei (anche nelle mense ospedaliere).

La diagnosi va condotta ricercando la/e tossina/e responsabile/i nelle feci e, soprattutto, negli alimenti incriminati. Per le spore di $C$. perfringens sono inoltre necessari dei conteggi particolari. In commercio è possibile, in parte, reperire kit specifici al riguardo.

\section{Agenti virali associati alle infezioni intestinali o gastro-intestinali}

Rotavirus, Adenovirus, Calicivirus, Astrovirus, e Norovirus (già SRSV, "Norwalk-like virus") sono causa frequente di diarrea con vomito, principalmente in bambini.

In tale sede tratteremo esclusivamente di Rotavirus e Adenovirus, per i quali esistono in commercio da tempo kit idonei per il loro riconoscimento, anche se recentemente sono comparsi kit per Norovirus ed Astrovirus, virus che sembrano essere tutt'altro che infrequenti e causa solitamente di forme epidemiche.

\section{Rotavirus}

Appartenenti alla famiglia Reoviridae virus a RNA a doppio filamento, sono causa di diarrea e vomito nei bambini più piccoli. Epidemie possono insorgere in asili nido. Occasionalmente $\mathrm{i}$ Rotavirus causano diarrea negli anziani. Le infezioni sembrano più frequenti nei periodi invernali. L'incubazione è di 1-3 gg.

Poiché i Rotavirus sono una delle cause più frequenti di diarrea nei bambini in tenera età, andrebbero sempre cercati quando vi è vomito, o in assenza dei 3 patogeni batterici più importanti. I metodi di ricerca dei Rotavirus nei campioni fecali prelevati nella fase acuta della malattia comprendono, nella diagnostica routinaria, metodi immunocromatografici, ELISA e l'agglutinazione con particelle di lattice.

\section{Adenovirus}

Gli Adenovirus, virus a doppia catena di DNA privi di envelope, sono implicati in molte infezioni pediatriche anche respiratorie e risultano in Europa forse secondi per importanza solo ai Rotavirus come causa di diarrea acuta nei bambini piccoli. Le epidemie sono state riscontrate negli asili nido e nei reparti pediatrici (anche per Rotavirus ciò è stato segnalato). Sono di frequen- te osservazione diarrea prolungata e, talora, rilievo termico moderato.

Per i metodi di ricerca valgono le medesime indicazioni fatte per i Rotavirus.

\section{PROCEDURE DIAGNOSTICHE}

\section{Diagnostica di base}

La procedura diagnostica minima, per la diarrea acuta autoctona (solitamente casi sporadici), prevede la ricerca di Salmonella, Shigella e Campylobacter. In bambini con vomito anche Rotavirus andrebbe sempre ricercato.

Le negatività per tali patogeni e la persistenza di diarrea dovrebbe indurre alla ricerca di $C$. difficile (tossina A) e di Adenovirus.

\section{Diagnostica allargata}

Per la ricerca di protozoi responsabili di diarrea (e parassiti in generale) si rimanda alle Linee Guida sopra ricordate, sebbene in molte circostanze la ricerca di alcuni protozoi patogeni più frequenti (Dientamoeba fragilis, Giardia duodenalis) o meno frequenti (Cryptosporidium spp) non andrebbe mai dissociata in un esame microbiologico delle feci.

Le situazioni in cui si deve ampliare lo spettro dei patogeni da ricercare sono deducibili da quanto esposto nelle premesse.

Negli schemi seguenti si puntualizzano alcune indicazioni operative, relativamente a determinati quadri nosologici, quali:

- diarrea protratta;

- diarrea nel paziente immunocompromesso;

- enterite epidemica in collettività;

- enterocolite emorragica;

- enterocolite da antibiotici/iatrogena/in pazienti ospedalizzati;

- proctocoliti nel maschio omosessuale;

- tossinfezioni alimentari;

- situazioni particolari. 


\begin{tabular}{|c|c|}
\hline QUADRO NOSOLOGICO & AGENTI MICROBICI DA RICERCARE \\
\hline $\begin{array}{l}\text { Diarrea acuta autoctona } \\
-\quad \text { sempre }\end{array}$ & $\begin{array}{l}\text { Salmonella } \\
\text { Shigella } \\
\text { Campylobacter }\end{array}$ \\
\hline - se vomito (in bambini) & Rotavirus \\
\hline $\begin{array}{ll}\text { - } & \text { se negativa } \\
\text { (con diarrea persistente) }\end{array}$ & $\begin{array}{l}\text { C. difficile (tossina A/B) } \\
\text { Adenovirus }\end{array}$ \\
\hline $\begin{array}{l}\text { Diarrea protratta } \\
\text { (oltre i I } 4 \text { giorni) }\end{array}$ & \begin{tabular}{|l|} 
Salmonella \\
Shigella \\
Campylobacter \\
Arcobacter butzleri \\
Protozoi \\
(C. difficile, tossina A/B)
\end{tabular} \\
\hline $\begin{array}{l}\text { Diarrea nel paziente } \\
\text { immunocompromesso }\end{array}$ & \begin{tabular}{|l|} 
Salmonella \\
Shigella \\
Campylobacter \\
Protozoi \\
\end{tabular} \\
\hline Diarrea del viaggiatore & 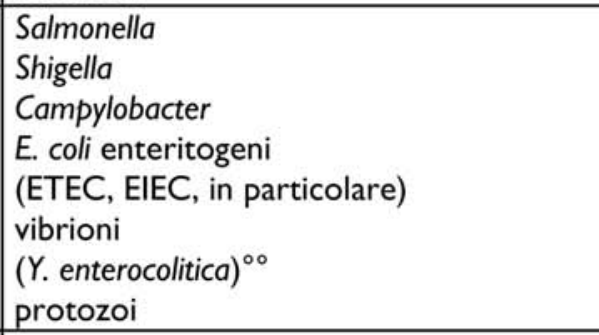 \\
\hline Enterite epidemica in collettività & $\begin{array}{l}\text { Salmonella } \\
\text { Campylobacter } \\
\text { (Shigella) }^{\circ \circ 0} \\
(\text { Rotavirus) } \\
\text { protozoi } \\
\left(\text { E. coli EPEC) }{ }^{\circ 000} \text { (in bambini <3anni) }\right.\end{array}$ \\
\hline Enterocolite emorragica & $\begin{array}{l}\text { E. coli VTEC (innanzitutto EHEC) } \\
\text { Campylobacter } \\
(\text { Salmonella) } \\
(\text { Shigella })^{\circ 00} \mathrm{e}^{\circ 000} \\
\end{array}$ \\
\hline $\begin{array}{l}\text { Enterocolite da antibiotici/iatrogena/in } \\
\text { Pazienti ospedalizzati }\end{array}$ & C. difficile (tossina A/B) \\
\hline Proctocoliti nel maschio omosessuale & \begin{tabular}{|l} 
Shigella \\
Salmonella \\
Campylobacter \\
Helicobacter cinaedi/H. fennelliae \\
Protozoi \\
Patogeni genitali
\end{tabular} \\
\hline Tossinfezioni alimentari & $\begin{array}{|ll|}\text { tossina S. aureus } & \text { (se possibile) }^{00000} \\
\text { tossina C. perfringens } & \text { (se possibile) } \\
\text { tossina B. cereus } & \text { (se possibile) }^{000000} \\
\text { Salmonella } & \\
\end{array}$ \\
\hline Situazioni particolari & Vedi PREMESSE \\
\hline
\end{tabular}

Note: ${ }^{\circ}$ se presente vomito; ${ }^{\circ}$ se proveniente da Paesi in cui è nota l'endemia; ${ }^{\circ 00}$ se ne è nota l'endemia;

${ }^{\circ 000}$ se negativi i precedenti; ${ }^{\circ 000}$ ricerca di tossina/e con kit specifici (se in dotazione; altrimenti inviare a centri specialistici al riguardo) 


\section{RACCOLTA, CONSERVAZIONE ED INVIO DEI CAMPIONI FECALI}

\begin{tabular}{|c|c|}
\hline Tempo ottimale di raccolta del campione & In fase acuta e prima possibile dopo l'esordio clinico \\
\hline Tipo di campione & Feci spontaneamente emesse \\
\hline Modalità di raccolta & $\begin{array}{l}\text { I campioni devono essere raccolti in una padella (o } \\
\text { superficie) asciutta e pulita e quindi trasferiti in } \\
\text { contenitori trasparenti, rigidi e a tenuta sicura. }\end{array}$ \\
\hline Quantità adeguata & 5 - 10 grammi; 5 - $10 \mathrm{ml}$ (se feci liquide) \\
\hline Numero appropriato di campioni & $\begin{array}{l}\text { I campione fecale è sufficiente. } \\
\text { Può rendersi necessario un secondo campione su } \\
\text { richiesta da parte del microbiologo }\end{array}$ \\
\hline Conservazione dei campioni e sicurezza operativa & $\begin{array}{l}\text { In contenitori rigidi (vedi sopra) a loro volta in } \\
\text { sacchetti di plastica chiusi (a doppia tasca: I per il } \\
\text { campione fecale, I per la scheda specifica) }\end{array}$ \\
\hline $\begin{array}{l}\text { Tempo tra la raccolta del campione e la } \\
\text { procedura analitica }\end{array}$ & $\begin{array}{l}\text { I campioni devono essere trasportati in laboratorio } \\
\text { quanto prima possibile (Shigella è particolarmente } \\
\text { labile); in ogni caso entro } 2 \text { ore (datando ora di } \\
\text { emissione). } \\
\text { Per Rotavirus/Adenovirus si possono conservare } \\
\text { overnight a } 2-6^{\circ} \mathrm{C} \text { (meglio se nella soluzione } \\
\text { tamponata prevista nel kit in uso). } \\
\text { Per qualsivoglia anomalia contattare il laboratorio. } \\
\text { Se è richiesto anche l'esame parassitologico } \\
\text { consultare le Linee Guida AMCLI-CoSP (Rif. } \\
\text { Bibliografico n. 8) }\end{array}$ \\
\hline Qualità e idoneità del campione & $\begin{array}{l}\text { Allegare sempre la scheda specifica (adeguatamente } \\
\text { compilata). }\end{array}$ \\
\hline
\end{tabular}




\section{PROCEDURE DIAGNOSTICHE PRELIMINARI}

\begin{tabular}{|c|c|}
\hline Valutazione macroscopica & $\begin{array}{l}\text { Aspetto: i campioni devono essere descritti come solidi } \\
\text { (non idonei, solitamente), non formati, liquidi o acquosi. } \\
\text { Segnalare la presenza di muco/sangue. }\end{array}$ \\
\hline Valutazione microscopica & $\begin{array}{l}\text { - } 40 \times 10 \text { al MO in SF: valutare leucociti, fagociti, sangue } \\
\text { occulto (in caso ricorre a test specifico). La presenza di } \\
\text { sangue si correla a enterocoliti/dissenterie batteriche (e } \\
\text { amebiche: la presenza di leucociti si correla a infezioni } \\
\text { batteriche (Campylobacter, Shigella, Salmonella); } \\
\text { Le infezioni da Adenovirus e Rotavirus (tranne casi } \\
\text { rarissimi) decorrono senza muco né sangue. } \\
-100 \times 10 \text { (immersione dopo colorazione di Gram): a } \\
\text { discrezione e in casi specifici (può essere utile per } \\
\text { Campylobacter); } \\
\text { - per parassiti: consultare le Linee Guida Operative di } \\
\text { AMCLI-CoSP (Rif. Bibliografico n. 8) }\end{array}$ \\
\hline $\begin{array}{l}\text { Procedura sul campione fecale: } \\
\qquad \text { tutti i batteri patogeni }\end{array}$ & $\begin{array}{l}\text { 1.Diffondere una piccola quota (alcune gocce) di } \\
\text { materiale fecale (anche risospeso in SF) su una piastra } \\
\text { per l'esame colturale, utilizzando un'area di circa I/3 - } \\
\text { 1/4 della superficie disponibile (si possono usare } \\
\text { bastoncini rigidi sterili). } \\
\text { 2. Diffondere l'inoculo con ansa sterile per ottenere } \\
\text { colonie isolate. } \\
\text { 3.Inoculare un volume di feci (in rapporto I:5 - I:9) in un } \\
\text { brodo di arricchimento. Dopo l'incubazione eseguire le } \\
\text { sottocolture utilizzando un'ansa sterile inserita per } \\
\text { raccogliere il campione al di sotto della superficie del } \\
\text { brodo e seminare su terreni appropriati. } \\
\text { Vedi punto } 2 \text {. }\end{array}$ \\
\hline $\begin{array}{l}\text { Procedura sul campione: } \\
\qquad \text { Campylobacter }\end{array}$ & $\begin{array}{l}\text { 1.Vedi sopra. } \\
\text { 2. Tecnica membrana filtrante (preferibile, soprattutto se } \\
\text { la ricerca è estesa a tutte le specie di tale genere e a } \\
\text { generi correlati): risospendere una parte delle feci in } \\
\text { brodo Brucella (o SF), meglio se preriscaldato; } \\
\text { omogeneizzare bene (p. es. con palline di vetro). } \\
3-4 \text { gocce vengono deposte sopra una membrana } \\
\text { filtrante sterile }(0.45-0.65 \mu \text { ) a sua volta appoggiata } \\
\text { sulla superficie di una piastra di agar sangue. Questa } \\
\text { piastra viene posta a } 37^{\circ} \mathrm{C} \text { in aerobiosi per } 45^{\prime} \text {, quindi, } \\
\text { dopo la rimozione della membrana, incubata a } 37^{\circ} \mathrm{C} \text { in } \\
\text { microaerofilia per } 24-48 \text { ore (per } \mathrm{C} \text {. jejuni/coli); sino a } 5 \\
\text { giorni per tutti gli altri. }\end{array}$ \\
\hline $\begin{array}{l}\text { Procedure sul campione: } \\
\text { Rotavirus / Adenovirus }\end{array}$ & Tecniche differenziate (vedi sistema in uso) \\
\hline $\begin{array}{l}\text { Procedure sul campione: } \\
\text { C. difficile (tossina A e B) }\end{array}$ & Tecniche differenziate (vedi sistema in uso) \\
\hline
\end{tabular}


TERRENI DI COLTURA, MICROORGANISMI E MODALITÀ OPERATIVE

\begin{tabular}{|c|c|c|c|c|c|}
\hline Microorganismi & $\begin{array}{c}\text { Terreni di } \\
\text { coltura }\end{array}$ & $\begin{array}{l}\text { Incubazione: } \\
\text { temperatura }\end{array}$ & $\begin{array}{c}\text { Incubazione: } \\
\text { atmosfera }\end{array}$ & $\begin{array}{c}\text { Incubazione: } \\
\text { tempi }\end{array}$ & $\begin{array}{l}\text { Lettura } \\
\text { colture }\end{array}$ \\
\hline $\begin{array}{l}\text { Salmonella } \\
\text { Shigella }\end{array}$ & $\begin{array}{l}\text { Agar } \\
\text { SS (o XLD o HEA) } \\
\text { e McConkey }\end{array}$ & $35-37^{\circ} \mathrm{C}$ & aerobiosi & $16-24$ ore & sup. 16 ore \\
\hline Campylobacter & $\begin{array}{l}\text { Campylobacter agar- } \\
\text { Selettivo } \\
\text { Agar sangue } \\
\text { (se membrana) }\end{array}$ & $\begin{array}{l}35-42^{\circ} \mathrm{C} \\
35-37^{\circ} \mathrm{C}\end{array}$ & $\begin{array}{l}\text { microaerofilia } \\
\text { microaerofilia }\end{array}$ & $\begin{array}{c}40-48 \text { ore } \\
18-48 \text { ore } \\
(5-7 \text { giorni) }\end{array}$ & $\begin{array}{l}\text { sup. } 40 \text { ore } \\
\text { sup. } 18 \text { ore }\end{array}$ \\
\hline Salmonella & $\begin{array}{l}\text { Brodo Selenite } \\
\text { (sub-coltura il } \\
\text { giorno dopo: } \\
\text { vedi sopra) } \\
\end{array}$ & $35-37^{\circ} \mathrm{C}$ & aerobiosi & $\begin{array}{l}8-24 \text { ore } \\
\text { (vedi sopra) }\end{array}$ & (vedi sopra) \\
\hline $\begin{array}{l}\text { E. coli } \\
\text { (tutti tranne EHEC) }\end{array}$ & Agar McConkey & $35-37^{\circ} \mathrm{C}$ & aerobiosi & $16-24$ ore & sup. 16 ore \\
\hline $\begin{array}{l}\text { E. coli - EHEC } \\
(O: 157)\end{array}$ & $\begin{array}{l}\text { Agar } \\
\text { McConkey- } \\
\text { sorbitolo }\end{array}$ & $35-37^{\circ} \mathrm{C}$ & aerobiosi & $16-24$ ore & sup. 16 ore \\
\hline Yersinia & Agar CIN & $28-30^{\circ} \mathrm{C}$ & aerobiosi & $16-24$ ore & sup. 16 ore \\
\hline Vibrio & $\begin{array}{l}\text { Agar TCBS } \\
+ \\
\text { Agar McConkey }\end{array}$ & $\begin{array}{l}35-37^{\circ} \mathrm{C} \\
35-37^{\circ} \mathrm{C}\end{array}$ & $\begin{array}{l}\text { aerobiosi } \\
\text { aerobiosi }\end{array}$ & $\begin{array}{l}16-24 \text { ore } \\
16-24 \text { ore }\end{array}$ & $\begin{array}{l}\text { sup. } 16 \text { ore } \\
\text { sup. } 16 \text { ore }\end{array}$ \\
\hline Aeromonas & $\begin{array}{l}\text { Aeromonas agar- } \\
\text { Selettivo } \\
\text { (o Agar McConkey) }\end{array}$ & $\begin{array}{l}28-30^{\circ} \mathrm{C} \\
28-37^{\circ} \mathrm{C}\end{array}$ & $\begin{array}{l}\text { aerobiosi } \\
\text { aerobiosi }\end{array}$ & $\begin{array}{l}16-24 \text { ore } \\
16-24 \text { ore }\end{array}$ & $\begin{array}{l}\text { sup. } 16 \text { ore } \\
\text { sup. } 16 \text { ore }\end{array}$ \\
\hline Plesiomonas & Agar McConkey & $35-37^{\circ} \mathrm{C}$ & aerobiosi & $16-24$ ore & sup. 16 ore \\
\hline $\begin{array}{l}\text { C. difficile } \\
\text { (in casi epidemici o } \\
\text { particolari) }\end{array}$ & $\begin{array}{l}\text { Agar CCFA } \\
\text { (o altro selettivo } \\
\text { specifico), } \\
\text { dopo shock alcolico } \\
\text { (in etanolo } \times \text { I ora) } \\
\text { o termico } \\
\left(80^{\circ} \mathrm{C} \times 10^{\prime}\right)\end{array}$ & $35-37^{\circ} \mathrm{C}$ & anaerobiosi & $40-48$ ore & sup. 40 ore \\
\hline
\end{tabular}


IDENTIFICAZIONI

\begin{tabular}{|c|c|c|}
\hline Livello minimo & $\begin{array}{l}\text { Salmonella } \\
\text { Shigella } \\
\text { Campylobacter } \\
\text { C. difficile } \\
\text { Rotavirus/Adenovirus }\end{array}$ & $\begin{array}{l}\text { A livello di genere } \\
\text { A livello di genere } \\
\text { A livello di genere } \\
\text { Tossina A/B } \\
\text { Kit commerciale }\end{array}$ \\
\hline Livello standard & $\begin{array}{l}\text { Salmonella } \\
\text { Shigella } \\
\text { Campylobacter/Arcobacter } \\
\text { E. coli EHEC (O:I57) } \\
\text { Yersinia enterocolitica } \\
\text { Vibrio } \\
\text { Aeromonas hydrophila } \\
\text { Plesiomonas shigelloides } \\
\text { C. difficile } \\
\text { Rotavirus/Adenovirus }\end{array}$ & $\begin{array}{l}\text { A livello di genere e sierogruppo } \\
\text { A livello di specie } \\
\text { A livello di specie } \\
\text { A livello di specie e sierotipo } \\
\text { A livello di specie } \\
\text { A livello di specie } \\
\text { A livello di specie } \\
\text { A livello di specie } \\
\text { Tossina A/B } \\
\text { Kit commerciale }\end{array}$ \\
\hline $\begin{array}{l}\text { Laboratorio di } \\
\text { Riferimento }\end{array}$ & $\begin{array}{l}\text { Salmonella } \\
\text { Shigella } \\
\text { Campylobacter/Arcobacter/Helicobacter } \\
\text { E. coli (tutti quando possibile) } \\
\text { Yersinia enterocolitica } \\
\text { Vibrio cholerae } \\
\text { Vibrio parahaemolyticus } \\
\text { Aeromonas hydrophila } \\
\text { Plesiomonas shigelloides } \\
\text { C. difficile } \\
\text { Bacillus cereus } \\
\text { Clostridium perfringens } \\
\text { Staphylococcus aureus }\end{array}$ & $\begin{array}{l}\text { A livello di specie/sierotipo } \\
\text { (in caso: fagotipo) } \\
\text { A livello di specie/sierotipo } \\
\text { (per S. sonnei, in caso: fagotipo) } \\
\text { A livello di specie } \\
\text { (tipizzazione in epidemie) } \\
\text { A livello di specie, sierotipo, test } \\
\text { tossina per VTEC } \\
\text { A livello di specie, sierotipo } \\
\text { A livello di specie, sierotipo } \\
\text { A livello di specie, sierotipo } \\
\text { A livello di specie } \\
\text { A livello di specie } \\
\text { A livello di specie, tossina A, tossina B } \\
\text { (tipizzazione in epidemie) } \\
\text { A livello di specie, sierotipo, tossina } \\
\text { A livello di specie, quantizzazione, } \\
\text { sierotipo, tossina } \\
\text { A livello di specie, tossina, fagotipo }\end{array}$ \\
\hline
\end{tabular}

\section{PROCEDURE DI REFERTAZIONE}

\begin{tabular}{|c|c|}
\hline Aspetto & Segnalare la presenza di muco/sangue. \\
\hline Esame microscopico & $\begin{array}{l}\text { Riportare la presenza di leucociti/fagociti (sangue occulto). } \\
\text { Riportare, se presenti, parassiti (per i quali vedi Linee Guida AMCLI } \\
\text { CoSP, Rif. Bibliografico n.8) }\end{array}$ \\
\hline Colture e tecniche differenziate & $\begin{array}{l}\text { Refertare la presenza o la assenza di patogeni specifici } \\
\text { (vedi Capitolo } 2 \text { PROCEDURE DIAGNOSTICHE). } \\
\text { Referto scritto: } 48-72 \text { ore (in caso sarà emesso un referto successivo). } \\
\text { Per i pazienti ospedalizzati comunicare immediatamente le positività. }\end{array}$ \\
\hline Prove di sensibilità & Refertare le sensibilità come clinicamente indicato. \\
\hline Annotazioni particolari & $\begin{array}{l}\text { Provvedere ad eventuali commenti circa il significato clinico, le } \\
\text { caratteristiche epidemiologiche, la terapia antibiotica, a discrezione del } \\
\text { medico microbiologo. }\end{array}$ \\
\hline
\end{tabular}




\section{BIBLIOGRAFIA}

1. Albert MJ, Faruque ASG, Faruque SM, Sack RB, Mahalanabis D. case-Control Study of Enteropathogens Associated with Childhood Diarrhea in Dhaka, Bangladesh. J Clin Microbiol 1999; 37: 3458-64.

2. Aleksic S, Bockemuel J. Yersinia and Other Enterobacteriaceae. In: Manual of Clinical Microbiology. Murray PR, Baron EJ, Pfaller MA, Tenover FC, Yolken RH Eds. American Society for Microbiology, 7th Ed., Whashington DC 1999: 48391.

3. Altwegg M. Aeromonas and Plesiomonas. In: Ibidem; 507-15.

4. Angulo J, Swerdlow DL. Bacterial infections in persons infected with Human Immunodeficiency Virus. Clin Infec Dis 1995; 21(suppl 1): 84-93.

5. Balakrish Nair G, Albert MJ, Shimada T, Takeda Y. Vibrio choleare 0139 Bengal: the new serogroup causing cholera. Rev Med Microbiology 1996; 7(1): 43 51.

6. Baron EJ, Peterson LR, Finegold SM. Bailey \& Scott' s Diagnostic Microbiology. Mosby Ed, $9^{\text {th }}$ ED, St. Louis MI 1994: 234-48.

7. Bartlett JG. Antibiotic-associated diarrhea. Clin Infec Dis 1992; 15: 573-81.

8. Bernieri F, Casella P, Crotti D, ed al. Linee Guida Operative per la diagnosi delle Parassitosi Intestinali. Microbiologia Medica 2005; 20(1): 39-46.

9. Bopp CA, Brenner FW, Wells JG, Strockbine NA. Escherichia coli, Shigella and Salmonella. In: Manual of Clinical Microbiology. Murray PR, Baron EJ, Pfaller MA, Tenover FC, Yolken RH Eds. American Society for Microbiology, $7^{\text {th }}$ Ed, Whashington DC 1999; 459-72.

10. Bourgault AM, Yechouron A, Gaudreau C, Gilbert H, Lamothe F. Should all stool specimens be routinely tested for Clostridium difficile? Clin Microbiol Infect 1999; 5: 219-23.

11. Butzler JP. New aspects in the diagnosis and treatment of infectious diarrhoea. G Ital Chemioter 1991; 38: 3-6.

12. Caprioli A, Crotti D, Luzzi I. Le Infezioni Gastroenteriche. Microb Med 2001, Numero Monografico AMCLI, volume 16, numero 1.

13. Caprioli A, Pezzella C, Morelli R, et al. Enteropathogens associated with childhood diarrhea in Italy. Pediatr Infect Dis J 1996; 15: 876-83.

14. Casemore DP, Roberts C. Guidelines for screening for cryptosporidium in stools: Report of a joint working group. J Clin Path 1993; 46(1): 2-4.

15. Chalmers RM, Salmon RL. Primary Care Surveillance for Acute Bloody Diarrhea, Wales. EID 2000; 6: 412-14.

16. Crotti D. Aspetti attuali nella diagnosi delle infezioni intestinali. La coprocoltura in chiave moderna. DOCUMENTA. Ed. Scientifiche Mascia Brunelli Biolife, Milano 1997.

17. Crotti D. Infezioni intestinali sostenute da Campylobacter jejuni/coli nella seconda metà degli anni '90: aspetti clinico-microbiologici e fenotipi di resistenza. GIMMOC 2002; Vol. VI Nº1: 19-24.

18. Crotti D. Parassitosi intestinali autoctone nella seconda metà degli anni '90: considerazioni critiche diagnostiche. Microb Med 2002; 17(1): 7-13.

19. Crotti D, D'Annibale ML. Dientamoeba fragilis e dientamoebosi: aspetti di parassitologia clinica e diagnostica di laboratorio. Parassitologia 2001; 43: 135-8.

20. Crotti D, D' Annibale ML, Fonzo G, Medori MC,
Ubaldi M. Diarre acute e protratte nella popolazione del territorio perugino: diagnosi microbiologica delle enteriti ed aspetti clinico-epidemiologici relativi al 2001. Le Infezioni in Medicina 2002; 2: 81-7.

21. Crotti D, Medori MC, D’Annibale ML, Fonzo G, Del Sante M. Infezione da Clostridum difficile in pazienti con diarrea nosocomiale e acquisita in comunità a Perugia. Microb Med 2001: 16(3): 313-5.

22. Crotti D, Medori MC, Fonzo G, Del Sante M, D'Annibale ML. Diarre in età pediatrica: l'infezione, la diagnosi, l'eziologia. Microb Med 1999; 14(4): 263-8.

23. Dehodhar LP, Sraswarthi K, Varudkar A. Aeromonas species and their association with human diarrheal disease. J Clin Microbiol 1991; 29(5): 853-6.

24. de Wit MAS, Koopmans MPG, Kortbeek M, et al. Gastroenteritis in Sentinel General Practices, the Nederlands. EID 2001; 7(1): 82-91.

25. Dionisi AM, Crotti D, Pezzotti G, et al. CAMPYG: sorveglianza delle infezioni da Campylobacter in Italia. Microb Med 2002; 17 (1): 58-63.

26. Eko FO, Rotimi VO. Vibrio parahaemolyticus in humans: disease spectrum, epidemiology and laboratori identification. Rev Med Microbiology 1995; 6(2): $137-45$.

27. Gascon J, Vargas M, Schellenberg D, et al. Diarrhea in Children under 5 Years of Age from Ifakara, Tanzania: a Case-Control Study. J Clin Microbiol 2000; 38: 4459-62.

28. Guerrant RL, Van Gilder T, Steiner TS, et al. Practice Guidelines for the Management of Infectious Diarrhea. CID 2001; 32: 331-51.

29. Guerrant RL, Steiner TS. Principles and syndromes of enteric infection. In. Mandell GL, Bennett JE, Dolin R, Principles and Practise of Infectious Diseases $\left(5^{\text {th }}\right.$ edition) New York 2000; 1076 - 1111.

30. Hedberg CW, et al. Changing epidemiology of foodborne disease: a Minnesota perspective. Clin Infect Dis 1994; 18: 671.

31. Kuroki S, Saida T, Nukina M, et al. Campylobacter jejuni strains from patients with Guillain-Barré syndrome belong mostly to Penner serogroup 19 and contain beta-N-acetylglucosamine residues. Ann Neurol 1993; 33: 243-7.

32. Liste MB, Natera I, Suarez JA, Pujol FH, Liprandi F, Ludert JE. Enteric Virus Infections and Diarrhea in Healthy and Human Immunodeficiency VirusInfected Children. J Clin Microbiol 2000; 38: 2873-7.

33. Lopez L, Castillo FJ, Fernadez MA, et al. Astrovirus Infection Among Children with Gastroenteritis in the City of Zaragoza, Spain. Eur J Clin Microbiol Infect Dis 2000; 19: 545-7.

34. Luzzi I, Covacci A, Censini S, et al. Detection of a Vacuolating Cytotoxin in Stools from Children with Diarrhea. CID 1996; 23: 101-06.

35. Miller JM, Holmes HT. Specimen Collection, Transport, and Storage. In: Manual of Clinical Microbiology. Murray PR, Baron EJ, Pfaller MA, Tenover FC, Yolken RH Eds. American Society for Microbiology, $7^{\text {th }}$ Ed. Washington DC 1999: 33-42.

36. Nachamkin I. Campylobacter and Arcobacter. In: Ibidem, 716-24.

37. Pedler SJ, Orr KE. Examination of faeces for bacterial pathogens. J Clin Pathol 1990; 43: 410-15.

38. Presterl E, Nadrchal R, Wolf D, Rotter M, Hirschl AM. Enteroaggregative an Enterotoxigenic Escherichia coli among Isolates from Patients with Diarrhea in Austria. Eur J Clin Microbiol Infect Dis 1999; 18: 209-12. 
39. Rossi S, Crotti D. Enterite acuta autoctona da Plesiomonas shigelloides. Microb Med 1999; 14(4): 341-2.

40. Schwaber MJ, Simhon A, Block C, Roval V, Federber N, Shapiro M. Factors Associated with Nosocomial Diarrhea and Clostridium difficile-Associated Disease on the Adult Wards of an Urban Tertiary Care Hospital.

41. Settle CD, Wilcox MH. Comparison of the Oxoid Clostridium difficile toxin A detection kit with cytotoxin detection by a cytopathic effect method examined at 4, 6, 24 and 48 h. Clin Microbiol Infect 1999; 5: 698-701.

42. Steiner TS, Amidou Samie, Guerrant RL. Infectious diarrhea: new pathogens and new challenges in developed and developing areas. Clin Infect Dis 2006; 43: 408-10.

43. Torres ME, Pirez MC, Schelotto F, et al. Etiology of Children's Diarrhea in Montevideo, Uruguay: Associated Pathogens and Unusual Isolates. J Clin Microbiol 2001; 39: 2134-9.

44. Tozzi AE, Caprioli A, Minelli F, et al. Shiga ToxinProducing Escherichia coli Infs Associated with Hemolytic Uremic Syndrome, Italy, 1988-2000. EID 2003; 9(1): 106-8.

45. Tozzi AE, Minelli F, Gorietti S, et al. Infezioni da VTEC in Italia, 1988-2000. Microb Med 2002; 17(1): 64-9.

46. Vargas M, Gascon J, Jmenez De Anta MT, Vila J. Prevalence of Shigella Enterotoxins 1 and 2 among Shogella Strains Isolated from Patients with Traveler's Diarrhea. J Clin Microbiol 1999; 37: 360811.

47. Vila J, Ruiz J, Gallardo F, et al. Aeromonas spp. and Traveler's Diarrhea: Clinical Features and Antimicrobial Resistance. EID 2003; 9(5); 552-5.

48. Wilhelmi I, Roman E, Sanchez-Fauquier A. Viruses causing gastroenteritis. Clin Microbiol Infect 2003; 247-62.

\section{Crotti Daniele}

Strada Comunale per Pilonico Paterno 4, 06080 Pianello, Perugia

Telefono: 075602372

E-mail: nenedc@tin.it 